\title{
ADAMTS13 missense variants associated with defective activity and secretion of ADAMTS13 in a patient with non-cirrhotic portal hypertension
}

\author{
Ashish Goel ${ }^{1}$, V.Raghupathy ${ }^{2}$, GJ Amirtharaj², Aaron Chapla ${ }^{3}$, Aparna Venkatraman ${ }^{4}$, Banumathi $^{2}$ \\ Ramakrishna $^{5}$, Anup Ramachandran ${ }^{2}$, Nihal Thomas $^{3}$, KA Balasubramanian ${ }^{2}$, Ian Mackie $^{6}$, Elwyn Elias $^{1,7}$, CE \\ Eapen $^{1}$ \\ Departments of Hepatology ${ }^{1}$, Wellcome Research Unit ${ }^{2}$, Endocrinology ${ }^{3}$, Stem Cell Research Center ${ }^{4}$ and Pathology ${ }^{5}$, \\ Christian Medical College, Vellore \\ Haemostasis Research Unit, Haematology Department, University College London, London, UK ${ }^{6}$; Emeritus Professor, \\ Liver Unit, University Hospital Birmingham, Birmingham, $\mathrm{UK}^{7}$
}

\begin{abstract}
Background: Non-cirrhotic intrahepatic portal hypertension (NCIPH), characterized by thrombotic microangiopathy of portal venous system, low ADAMTS13 (a disintegrin like and metalloproteinase with thrombospondin type 1 motifs-13) and high vWF (von-Willebrand factor) levels. This study aimed to screen for ADAMTS13 mutations, focusing on the CUB domain, in these patients.
\end{abstract}

Methods: Prospectively recruited NCIPH patients and healthy volunteers underwent tests for plasma vWF-ADAMTS13 balance. Sanger sequencing of CUB domain of ADAMTS13 was done in a subset of the NCIPH patients and the detected mutation was screened for in all the study participants. Next generation sequencing of clinically relevant exome and liver immunostaining for ADAMTS13 was done in patient with detected ADAMTS13 mutation.

Results: Plasma vWF-ADAMTS13 balance was significantly altered in 24 NCIPH patients (Child's class $\mathrm{A}: 23, \mathrm{~B}: 1)$ as compared to 22 controls. On initial sequencing of the CUB domain (17 cases and 3 controls), one NCIPH patient showed a rare mis-sense variant (SNV) at position c.3829C $>$ T 
resulting in p.R1277W (rs14045669). Subsequent RFLP analysis targeted to R1277W variant, did not detect this in any other NCIPH patient, nor in any of the 22 controls.

NCIPH patient with R1277W variant, had severe ADAMTS13 deficiency, consistently high vWF, other mis-sense SNVs in ADAMTS13, vWF and complement genes. Immuno-staining of his liver biopsy revealed globules of ADAMTS13 within stellate cells.

Conclusions: We report mis-sense variants in ADAMTS13, vWF and complement genes in a patient with NCIPH who had decreased secretion and activity of ADAMTS13 protein. Further studies are needed in NCIPH patients in this regard.

Keywords : Non-cirrhotic portal fibrosis; microangiopathy; liver; next generation sequencing; complement system 


\section{Introduction}

Idiopathic non-cirrhotic intrahepatic portal hypertension (NCIPH) is characterised by occlusion of small intrahepatic portal vein radicles. Studies from India have shown that NCIPH is an ongoing problem especially in lower socioeconomic strata $[1,2]$. We have demonstrated ADAMTS13 (a disintegrin like and metalloproteinase with thrombospondin type 1 motifs - 13) deficiency in patients with NCIPH despite preserved liver function [3, 4]. ADAMTS13, a vWF (von-Willebrand factor) cleaving protease secreted mainly from the hepatic stellate cells [5] and P-selectin, a protein anchoring vWF to endothelial surface are involved in primary hemostasis. In this study, we studied vWF-ADAMTS13 balance in these patients.

ADAMTS13 gene is located on chromosome 9q34 (29 exons). ADAMTS13 protein consists of a number of domains including the metalloprotease domain (responsible for cleaving) at the $\mathrm{N}$ terminal and multiple CUB (complement $\mathrm{C} 1 \mathrm{r} / \mathrm{C} 1 \mathrm{~s}$, urinary epidermal growth factor, and brain natriuretic peptide-1) domains at the C-terminal, which play important role in the binding of the protein to its substrate [6].

We have noted lower plasma ADAMTS13 activity in NCIPH patients when the ADAMTS13 activity was assayed using the complete vWF molecule as a substrate (i.e. collagen binding assay) compared to the activity using a synthetic vWF peptide substrate that contains the cleavage site (i.e. FRET or Fluorescence resonance transfer assay) [3]. We postulated that this phenomenon reflected defective binding of ADAMTS13 to vWF, a prerequisite for cleavage [7]. Mutations in the CUB domain of ADAMTS13 are associated with decreased activity and also defective secretion of ADAMTS13 from hepatic stellate cells [8]. In this study, we looked for mutations in the CUB domain of the ADAMTS13 gene (exons 26 and 27) in NCIPH patients as compared to healthy controls. 
The alternative complement pathway is activated by decreased expression of ADAMTS13 [9]. Unrestrained activation of alternative complement pathway is one of the mechanisms involved in the pathogenesis of thrombotic microangiopathy [9,10]. Localized intra-hepatic complement activation may also play a role in the pathogenesis of NCIPH. Over-activation of complement pathway is associated with familial mutations in genes involved in alternative/ lectin complement pathways. Recent reports describing mutations in KCNN3 [11] and DGUOK [12] genes in familial NCIPH patients reaffirm the pathogenic role of genetic factors in NCIPH.

In this study, we carried out an evaluation for ADAMTS13- CUB domain mutations in a cohort of NCIPH patients. In a single NCIPH patient with ADAMTS13 CUB domain mutation, detailed genetic analysis (performed by next generation sequencing of clinically relevant exome) of ADAMTS13, vWF, alternative complement pathways and KCNN3/ DGUOK was performed.

\section{Methods :}

Objective 1 : Hospital based case-control study was performed to assess prevalence of mutation(s) in CUB domain of ADAMTS13 gene and also assess vWF-ADAMTS13 imbalance and plasma Pselectin in NCIPH patients as compared to healthy volunteers.

Objective 2 : In a NCIPH patient with ADAMTS13 CUB domain mutation, we performed the following tests :

a. clinical exome sequencing using a custom capture kit to achieve high sequencing coverage for the target genes of interest. These genes included ADAMTS13 $(37 \mathrm{~Kb})$, vWF (176 Kb), alternative complement pathway genes [CFH (95 Kb), CFI (61 Kb), CFB (24 Kb), C3 (53 $\mathrm{Kb}), \mathrm{CD} 46(43 \mathrm{~Kb})]$, lectin complement pathway gene $\{\mathrm{MBL} 2(6.3 \mathrm{~Kb})\}$ and genes recently described in familial NCIPH $\{$ KCNN3 $(173 \mathrm{~Kb})$ and DGUOK $(32 \mathrm{~Kb})\}$. 


\section{Case recruitment :}

Consecutive NCIPH patients visiting liver clinic were recruited as cases. Healthy volunteers served as healthy controls. NCIPH was diagnosed as per published criteria - all patients had gastroesophageal varices on endoscopy, patent portal vein and hepatic venous outflow tract on ultrasound doppler, an unyielding detailed evaluation for known etiologies of liver disease and liver biopsy showing the absence of advanced fibrosis and absence of findings suggesting alternate etiology, e.g. autoimmune hepatitis etc. Diseases, known to histologically mimic NCIPH (e.g. sarcoidosis, schistosomiasis etc.) were excluded [3].

24 NCIPH patients were recruited for the study. The route of liver biopsy was - transjugular : 9 patients; percutaneous : 7; per-operative : 8 \{tru-cut and wedge biopsies of liver obtained during splenorenal shunt surgery (7) and splenectomy (1)\}. On transjugular liver biopsy, 3 (2-7); median (range) cores of the liver with maximum size 11.5 (3-13) $\mathrm{mm}$ and containing 7 (5-13) portal tracts were obtained. Similarly, on percutaneous liver biopsy 2 (1-3) cores of the liver of maximum size 15.5 (10-19) $\mathrm{mm}$ and containing 7 (5-19) portal tracts were obtained. On liver biopsy, 14 patients had mild portal fibrosis and 4 had no portal fibrosis, 5 had focal bridging fibrosis.

Besides evaluation of the severity of liver disease in patients with NCIPH, all study participants underwent assays to determine the plasma ADAMTS13-vWF balance, plasma P-selectin (protein anchoring vWF to the endothelial surface) and also evaluation for mutation in CUB domain of ADAMTS13 gene. 


\section{Assays of plasma levels of ADAMTS13, vWF and P-selectin in a cohort of NCIPH patients :}

Plasma levels of ADAMTS13, vWF and P-selectin were tested on citrated platelet poor plasma stored at $-80^{\circ} \mathrm{C}$ until assay. ADAMTS13 FRET activity, vWF antigen/ acticity, and P-selectin concentrations were estimated by commercially available kits from American Diagnostica Inc, Hyphen BioMed and R\&D Systems respectively.

ADAMTS13 activity was assessed by two methods- fluorescence resonance energy transfer (FRET) method which measures the increase in fluorescence when ADAMTS13 uncouples the recombinant -VWF86 ALEXA FRET substrate; and also by measuring residual collagen binding activity of purified vWF concentrate (in-house collagen binding assay, CBA) as was previously described [13]. The manufacturer's normal ranges for plasma ADAMTS13 antigen, ADAMTS13 FRET activity, vWF antigen, and P-selectin were: $740 \pm 110 \mathrm{ng} / \mathrm{ml}, 50 \%-150 \%, 0.5-1.5 \mathrm{IU} / \mathrm{ml}$ and $18-40 \mathrm{ng} / \mathrm{ml}$ respectively. vWF antigen and ADAMTS13 antigen were converted to a percentage of normal plasma for calculation of vWF:ADAMTS13 ratio.

\section{Sequencing the CUB domain of ADAMTS13 gene in a cohort of NCIPH patients :}

Genomic DNA was isolated from blood of $17 \mathrm{NCIPH}$ patients as well as from 3 healthy controls using QIAGEN DNA extraction kit as described by the manufacturer. Genomic DNA (100ng) was utilized to amplify exons 26 and 27 pertaining to CUB domain of ADAMTS13 gene (9q34). The PCR reaction mixture $(50 \mu \mathrm{l})$ containing $25 \mu \mathrm{l}$ Taq DNA polymerase, 2x Master Mix Red, and forward (5' ACA GGG TCC ACC CCT ACC T 3') and reverse (5' CCC AGT TCC TTC CAT CTC AG 3') primers (4 pmol each) were used as described previously. The PCR conditions were: Initial denaturation at $95^{\circ} \mathrm{C}$ for 2 min followed by 35 cycles of $95^{\circ} \mathrm{C}$ for $30 \mathrm{~s}, 56^{\circ} \mathrm{C}$ for $30 \mathrm{~s}, 72^{\circ} \mathrm{C}$ for 1 min and final extension at $72^{\circ} \mathrm{C}$ for $5 \mathrm{~min}$. The successful amplification was evident by visualization of a specific $901 \mathrm{bp}$ PCR product on agarose gel electrophoresis after staining with ethidium bromide. 
Purified PCR products (901 bp) was sequenced both in the sense and anti-sense orientation using the Big Dye Terminator V3.1 cycle sequencing kit in a 3130 Genetic Analyzer from AB Applied Biosystems. The sequence was analyzed for mutations using the NovoSNP software.

\section{RFLP for the R1277W mutation in a cohort of NCIPH patients :}

RFLP for the detected ADAMTS13-CUB domain variant (R1277W) was performed in all 24 NCIPH patients and 22 healthy controls. PCR products were digested with the restriction endonuclease BalI to identify R1277W variant. Purified PCR product ( $1 \mu \mathrm{g})$ was digested by BalI (2 units) at $37^{\circ} \mathrm{C}$ for 2 hours and the digested products were separated by agarose gel (2\% (W/V)) electrophoresis.

Clinical Exome Sequencing and immunostaining of liver biopsy for ADAMTS13 in one NCIPH patient with ADAMTS13 R1277W variant

To preclude other causative mutations, clinical exome sequencing was carried out in one NCIPH patient (who had ADAMTS13 R1277W variant). Exome capture was performed on 50ng of genomic DNA sample using Nextera Rapid Capture custom enrichment. The library was sequenced at a mean coverage of $>80-100 \mathrm{X}$ on Illumina sequencing platform. The sequences obtained were aligned to the human reference genome (GRCh37/hg19) using BWA tool and the variant calling was performed using GATK-Lite based tools. Further, the annotation of variants identified was carried out against the Ensemble release 75 gene model. $98 \%$ of the coding region of ADAMST13 was covered by the sequencing process with $96.1 \%$ of the reads of quality $\geq$ Q30. Further, validation of the sequencing data analysis to identify variations in target genes of interest pertaining to ADAMTS13-vWF axis (ADAMTS13, vWF), alternative/ lectin complement pathways (CFH, $C D 46, C F I, C F B, C 3, M B L 2)$ and genes recently described associated with familial NCIPH ( $K C N N 3$ 
and $D G U O K$ ) was carried out using DNA Star software (DNASTAR, Inc. Madison, Wisconsin, USA).

Indirect immunofluorescence technique was used to immunostain the liver biopsy of this NCIPH patient using a commercial rabbit polyclonal antibody to ADAMTS13 (H-300, Santa-Cruz Biotechnology, Inc). The nuclei were labelled by 4',6-diamidino-2-phenylindole(DAPI) and stellate cells were stained with glial fibrillary acidic protein (GFAP).

\section{Statistical analysis :}

Variables were expressed as numbers (percentage) or median (range) as appropriate. Nonparametric tests (Mann-Whitney U, Wilcoxon signed ranks test, Fisher exact test or Chi-square test) were used for comparison and a $p$-value of $\leq 0.05$ was considered as statistically significant. The study was approved by the institutional ethics committee.

\section{Results}

Twenty four NCIPH patients and 22 healthy controls were recruited for this study. Table 1 depicts the regional distribution, demographics and baseline characteristics of the study participants. All NCIPH patients were in Child's class A (23 patients) or B (1 patient). There was no family history of liver disease in NCIPH patients.

Twenty one of the 24 NCIPH patients had thrombocytopenia - severe $(<50000 / \mathrm{cmm}): 6$; moderate $(50,000 / \mathrm{cmm}-75,000 / \mathrm{cmm}): 4$; and mild $(75,000 / \mathrm{cmm}-1,50,000 / \mathrm{cmm}): 11$. Three of the $20(15 \%)$ patients tested had low serum vitamin B12 levels $(<200 \mathrm{pg} / \mathrm{ml})$. Hepatic venous pressure gradient measured in $11 \mathrm{NCIPH}$ patients was 7 (5-18) $\mathrm{mm} \mathrm{Hg}$; median (range). In two patients, HVPG was $\leq 5 \mathrm{~mm} \mathrm{Hg}$. One NCIPH patient each had associated celiac disease and arsenicosis. 


\section{Plasma ADAMTS13-vWF levels in cohort of NCIPH patients and in controls :}

vWF antigen in $23 \mathrm{NCIPH}$ patients $(1.8,1-5.3 \mathrm{IU} / \mathrm{ml}$; median, range) was significantly higher than in 18 healthy controls $(0.9,0.4-1.66 \mathrm{IU} / \mathrm{ml}$; p-value <0.001); figure 1.

Plasma ADAMTS13 antigen in $15 \mathrm{NCIPH}$ patients $(486,239-614 \mathrm{ng} / \mathrm{ml}$; median, range) was significantly lower than in 18 healthy controls $(753,394-107 \mathrm{ng} / \mathrm{ml}$; p-value<0.001). Figure 1 also depicts the deranged vWF:ADAMTS13 ratio in NCIPH patients $(2.4,1.2-6.5)$ as compared to healthy controls $(0.9,0.5-1.8$; p-value: $<0.001)$.

Figure 2 depicts the plasma ADAMTS13 activity as assayed by FRET and collagen binding assays in NCIPH patients $(76 \%, 39-136 \%$ and $66 \%, 10 \%-105 \%)$ as compared to healthy controls (110\%, $63 \%-161 \%$ and $104 \%, 83 \%-122 \%$ ) respectively. In both these assays, the ADAMTS13 activity was significantly lower in NCIPH patients (p-value: 0.008 and p-value <0.001) as compared to healthy controls. In NCIPH patients, there was a trend to lower ADAMTS13 activity by collagen binding assay (CBA) as compared to ADAMTS13 activity by FRET assay.

One NCIPH patient (Child's score : 6) had severe ADAMTS13 deficiency (ADAMTS13 activity by CBA <10\%) and six NCIPH patients (Child's score: 5 in four patients and Child's score: 6 in 2 patients) had a mild ADAMTS13 deficiency (ADAMST13 activity by CBA $\leq 55 \%$ ). None of the healthy controls had deficient ADAMTS13 activity by CBA.

\section{P-selectin levels in cohort of NCIPH patients and in controls}

Plasma P-selectin levels were similar in $15 \mathrm{NCIPH}$ patients (39 ng/ml, 5-144 ng/ml; median, range) and 9 healthy controls (34 ng/ml, 8-41 ng/ml; p-value : 0.5). 7/15 NCIPH patients had high plasma P-selectin levels, i.e. $>40 \mathrm{ng} / \mathrm{ml}$ as compared to $1 / 9$ healthy controls (p-value : 0.2 ). 


\section{Mutation analysis in the CUB domain of ADAMTS13 gene in cohort of NCIPH patients}

Sequencing of the CUB domain was done in $17 \mathrm{NCIPH}$ patients and 3 healthy controls. One NCIPH patient showed a rare nucleotide change at position c.3829C $>\mathrm{T}$ resulting in $\mathrm{p} . \mathrm{R} 1277 \mathrm{~W}$ (rs14045669).

We further performed BalI RFLP analysis targeted to R1277W variant detection in all 24 NCIPH patients and 22 healthy controls (including the subjects in whom sequencing was done). Except for one NCIPH patient with R1277W variant on sequencing, no other subjects had this variant, figure 3.

Clinical exome sequencing variant analysis in one NCIPH patient with ADAMTS13 R1277W variant :

No reported pathogenic variants were identified by clinical exome sequencing in this patient. However, further analysis of the clinical exome sequence data for the target genes of interest detected the presence of several nucleotide changes including heterozygous missense variants (p.R1277W \& p.Q448E) in ADAMTS13 gene and also in CFI, CFB, CFH and $v W F$ genes (Table 2). All observed missense variants were predicted to be polymorphisms or benign by bio-informatic tools Polyphen-2, SIFT and Mutation taster (www.mutationtaster.org). Only R1277W variant (dbSNP rs140450669) in ADAMTS13 gene was found to be rare and was reported in ExAc database (Exome Aggregation consortium) with an allelic frequency of $0.017 \%$ in South Asians and $0.002 \%$ in Europeans. Details of the non-synonymous variants are given in Table 2. The list of all variants in the genes of interest is provided as supplementary material.

Immunostaining of liver biopsy in one NCIPH patient with ADAMTS13 R1277W variant :

On immunostaining of normal liver tissue, we demonstrated co-localisation of ADAMTS13 and GFAP (which stains liver stellate cells,). On studying the ADAMTS13 immuno-stained liver biopsy 
slides, on light microscopy, unlike normal/ cirrhotic control, more punctate and diffuse ADAMTS13 staining, and under high power, globules of ADAMTS13 were seen in stellate cells (Figure 4).

\section{Clinical details of NCIPH patient with ADAMTS13 R1277W variant:}

21 year old male, born to non-consanguineous parentage, belonging to lower socioeconomic class (as assessed by modified Kuppuswamy score), residing in northern India presented with splenomegaly since 10 years of age and hematemesis at the age of 17 years. He had no family history of similar illness. He underwent proximal splenorenal shunt surgery in 2012. Per-operative liver biopsy showed mild portal fibrosis. He developed glomerulonephritis (renal biopsy suggesting C3 nephritis) 15 months after the shunt surgery and was treated unsuccessfully with steroids. He developed secondary portal vein thrombosis and had worsening kidney function on follow-up. He suffered from two episodes of spontaneous bacterial peritonitis and subsequently was on maintenance hemodialysis from 2.5 years after the shunt surgery. Repeat kidney biopsy revealed diffuse, proliferative sclerosing glomerulonephritis with deposits of $\operatorname{IgA}, \operatorname{IgG}, \operatorname{IgM}$ and C3.

He had low ADAMTS13 activity (lowest level CBA:10\%; FRET : 68.3\%) and high vWF antigen levels at the initial visit $(5.33 \mathrm{IU} / \mathrm{ml})$ in the plasma. The subsequent ADAMTS13 levels were within normal range, but vWF activity assessed by collagen binding assay remained high $\{2.9 \times \mathrm{XLN}$ (2.63.5xULN); median, range\}, Table 3. Plasma P-selectin levels were high (100 ng/ml). He had platelet counts in the normal range (post-splenectomy status) and had no features suggesting microangiopathy (like schistocytes in peripheral blood smear) in the systemic circulation. Total complement activity was low (60\%, normal: 80-96\%) with low C3 (76 mg/dl, normal: 90-180 $\mathrm{mg} / \mathrm{dl})$ and preserved C4 (11.4 mg/dl, normal: 10-140 mg/dl); suggesting possible activation of the alternative complement pathway. There was no inflammation on duodenal biopsy and anti-tissue 
transglutaminase antibody was negative. There were no skin changes suggesting arsenicosis, but nail arsenic levels were not tested.

\section{Discussion :}

NCIPH is a relatively common cause of unexplained portal hypertension in India [1,2]. Our two previous reports from India and UK have demonstrated low ADAMTS13 activity, especially with collagen binding activity assay, in NCIPH patients despite preserved liver function. The present study also reiterates this finding, with all three studies demonstrating lower activity with ADAMTS13-CBA assay (activity against the complete vWF molecule) compared to ADAMTS13-FRET assay (activity against vWF peptide fragment having the cleaving site). This suggests relatively preserved specific vWF cleaving activity of ADAMTS13, prompting us to embark on a study to assess exons pertaining to CUB domain (thought to be responsible for the initial binding of ADAMTS13 to vWF) of ADAMTS13 gene.

In this study, of the $24 \mathrm{NCIPH}$ patients, we found mutations in the CUB domain of ADAMTS13 in a single patient. This patient had severely deficient ADAMTS13 collagen binding activity (on single occasion), consistently raised vWF and retention of ADAMTS13 globules in the hepatic stellate cells. Immunostaining of liver biopsy strongly suggests a defect in ADAMTS13 secretion. His plasma vWF activity, on four occasions, was elevated 2.6-3.5 times the upper limit of normal. Hence, this rare variant (p.R1277W) in exon 27, pertaining to CUB domain of ADAMTS13 gene might have a role in defective ADAMTS13 protein secretion which has to be functionally validated. Deletion of exon 27 (pertaining to CUB domain) of ADAMTS13 has also been noted in one patient with hereditary TTP [14]. Neither the other NCIPH patients nor the healthy controls had any detectable mutation in the 
ADAMTS13 CUB domain. The functional significance of this mutation needs to be further characterised.

In order to understand the possibility of other genes involvement in NCIPH, we performed clinical exome sequencing in this NCIPH patient with ADAMTS13 CUB domain (p.R1277W) mutation. Another missense variant in ADAMTS13 gene Q448E (rs2301612; cysteine rich region) was also identified in this patient. This SNP has been previously reported in families with congenital thrombotic thrombocytopenic purpura (TTP) [15]. Allele frequency of rs2301612 was 19\% (tested in 364 healthy subjects from Japan [16]). The frequency of this polymorphism in Indian population needs to be characterised. Though in the study by Kokame et al [15], no functional impact of this mutation (rs2301612) was noted on vWF cleaving activity, it has been shown that along with other ADAMTS13 mutations it can modulate ADAMTS13 protein expression and activity [17]. This variation has been shown to have a deleterious impact on other vascular pathology e.g. cerebral aneurysms [17].

Altered vWF-ADAMTS13 balance is noted in advanced stages of cirrhosis and may be involved in the progression of disease [18-20]. In NCIPH patients we have noted deficient ADAMTS13 activity in patients with preserved liver function, suggesting a role in the pathogenesis of intrahepatic portal venous occlusion $[3,4]$.

Table 2 lists the various non-synonymous variants noted in the NCIPH patient on targeted exome sequencing of the genes of interest (pertaining to ADAMTS13-vWF, alternative and lectin complement pathways, DGUOK and KCNN3). Besides R1277W variation, there were rare variants (minor allele frequency of $<0.5 \%$ [21]) noted in alternative complement pathways (CFI and $\mathrm{CFH}$ ). Though none of these variants have previously been associated with NCIPH, the effect of combined burden of these variants is unclear. It is expected that rare variants are more likely to confer risks for a disease process and same needs to be further studied [22]. 
The other variants in CFH and CFB (rs1061170 and rs12614 respectively) have been previously reported with uncertain significance in atypical haemolytic uremic syndrome, another form of thrombotic microangiopathy. Previous studies also describe other variations in complement genes in patients with congenital TTP and haemolytic uremic syndrome [23]. Acquired or inherited deficiency of ADAMTS13 results in accumulation of ultra-large vWF multimers, which in turn predisposes platelet adhesion to the endothelium [24-26]. ADAMTS13 deficiency is implicated in TTP and its role in various other thrombotic conditions is under study $[27,28]$. Genetic variations in ADAMTS13 can result in inherited form of severe TTP (Upshaw Schulman Syndrome) or may result in partial ADAMTS13 activity loss predisposing to various thrombotic events [8,29-32].

ADAMTS13 deficiency may trigger complement activation in patients with thrombotic microangiopathy [9]. In addition to low ADAMTS13, activation of alternative complement pathway is an important mechanism for thrombotic microangiopathy.

Also, there were recent reports of single gene mutations in DGUOK [12] and KCNN3 [11], implicated in familial NCIPH inherited in autosomal recessive/ dominant manner respectively. We did not find any variations in these genes in a single NCIPH patient.

Unlike the autosomal dominant/ recessive inheritance noted in some of the studies, none of our NCIPH patients in this study had a family history of similar liver disease. This suggests that NCIPH, in most patients, is a complex disorder, where a genetically predisposed individual when challenged by an environmental risk factor, develops localised portal vasculopathy. Thus, either a shared genetic predisposition or a shared environmental insult could be responsible for the increased prevalence of NCIPH in India and other developing countries [33]. As the pathology is limited to portal microcirculation, in previous studies we looked at the role of gut in the pathogenesis of NCIPH. There was an increased prevalence of non-specific gut inflammation [34], celiac disease, and gut derived toxins (arsenicosis) [35] 
in these patients. In the present study, one NCIPH patient each had arsenicosis and celiac disease.

As in our previous report [36], in this study also $15 \%$ of NCIPH patients had low vitamin B12 levels. In India, with endemic vitamin B12 deficiency, presence of vitamin B12 deficiency in patients with unexplained portal hypertension is a marker of absence of advanced fibrosis and NCIPH [37]. It is unclear if this is association has any causative implications in pathogenesis of NCIPH.

Worsening vWF-ADAMT13 imbalance is noted with advancing stages of cirrhosis $[19,20]$. This may contribute to focal parenchymal extinction in the liver noted in these patients and consequently important in the progression of disease [38]. Altered vWF-ADAMTS13 balance may also play a role in pro-thrombotic mileu noted in cirrhotics [39]. There is scope for wide ranging therapeutic potential targeting the vWF-ADAMTS13 imbalance to treat NCIPH in particular and portal hypertension in general [40].

Thus, in our study we demonstrate missense variants in ADAMTS13, vWF and complement genes in a single patient with NCIPH, associated with decreased secretion and activity of ADAMTS13 protein. Further research in this area is warranted. 


\section{Compliance with ethical standards}

Conflict of interest: AG, VR, GJA, AC, AV, BR, AR, NT, KAB, IM, EE and CEE confirm

that they have no conflicts of interest to declare.

Ethics statement : The study was performed in a manner to conform with the Helsinki Declaration of 1975, as revised in 2000 and 2008 concerning human and animal rights, and the authors followed the policy concerning informed consent as shown on Springer.com.

Financial support : The authors acknowledge the funding from Department of Science and technology, Govt. of India (SERB: EMR/2015/000570) and Fluid research fund from Christian Medical College, Vellore.

Acknowledgements : The authors acknowledge the support of Dr. Shalimar (Gastroenterology, AIIMS, N. Delhi) for making available the previous medical records. 


\section{Figure legends :}

Figure 1: vWF (panel A), ADAMTS13 (panel B) antigen and vWF:ADAMTS13 ratio (panel C) in patients with NCIPH as compared to healthy controls. The horizontal line in panel A and B depicts the upper normal limit of vWF and lower normal limit of ADAMTS13 antigen respectively.

N.B: vWF concentrations were available in $15 \mathrm{NCIPH}$ patients and 18 healthy controls, ADAMTS13 concentrations were available in $23 \mathrm{NCIPH}$ patients and 18 healthy controls.

Figure 2 : Plasma ADAMTS13 activity assayed by FRET (fluorescence resonance energy transfer) assay and collagen binding assay (CBA) in NCIPH patients as compared to healthy controls. The ADAMTS13 values for the patient with R1227W mutation in ADAMTS13 CUB domain are marked with asterisk $(*)$.

N.B: FRET available in $23 \mathrm{NCIPH}$ patients and 18 healthy controls, CBA available in $20 \mathrm{NCIPH}$ patients and 13 healthy controls.

Figure 3: Restriction fragment length polymorphism on the ADAMTS13 CUB domain PCR product (901 bp). The mutation R1227W created a new restriction site for DNA restriction enzyme BalI, resulting in extra bands in one NCIPH patient

Figure 4 : Indirect immunofluorescence of liver biopsy. Panel A and B shown co-localisation of stellate cell marker GFAP (white) and ADAMTS13 (red) in normal liver. Panels C and F (normal), panels D and G (cirrhotic control) and panels E and H (NCIPH patient with R1277W mutation) show more diffuse and punctate staining as well as ADAMTS13 globules in the higher magnification in NCIPH patient. Similar globules were not seen in normal/ cirrhotic liver biopsy. 
Table 1 : Demographics and baseline disease status in study participants.

\begin{tabular}{|l|l|l|}
\hline Parameters $^{\#}$ & $\begin{array}{l}\text { NCIPH } \\
(\mathbf{n = 2 4})\end{array}$ & $\begin{array}{l}\text { Healthy controls } \\
(\mathbf{n = 2 2})\end{array}$ \\
\hline Age (years) & $37(17-66)$ & $33(28-61)$ \\
\hline Sex (M:F) & $20: 4$ & $17: 3$ \\
\hline Regional distribution (E:S:W:N:F)* & $9: 11: 0: 1: 3$ & $1: 18: 0: 1: 0$ \\
\hline Serum bilirubin (mg\%) & $1(0.3-4.9)$ & - \\
\hline Serum Albumin (mg\%) & $3.8(2.5-4.9)$ & - \\
\hline Serum Creatinine (mg\%) & $0.9(0.6-2.5)$ & - \\
\hline International Normalised Ratio & $1.1(1-1.2)$ & - \\
\hline Child's score & $5(5-7)$ & - \\
\hline Hemoglobin $(\mathbf{g \%})$ & $11.9(8.4-14.6)$ & - \\
\hline Platelet count $(\mathbf{x 1 0}$ / cmm) & $0.9(0.05-2.33)$ & - \\
\hline Serum Vitamin B12 $\mathbf{~ p g / m l ~ ( n = 2 0 ) ~}{ }^{@}$ & $411(150-1110)$ & - \\
\hline
\end{tabular}

\#All values are depicted as numbers or median (range); *Regional distribution :: E: Eastern India; S : Southern India; W: Western India; N: Northern India; F: Foreign nationals; all from Bangladesh. @ Serum Vitamin B12 normal range: 200-950pg/ml; 3 patients had $<200$ pg/ml. 
Table 2: List of non-synonymous variants in the targeted sequencing of the genes of interest

\begin{tabular}{|c|c|c|c|c|c|c|c|c|}
\hline Gene & DNA Change & $\begin{array}{c}\text { Amino Acid } \\
\text { Change }\end{array}$ & dbSNP ID & $\begin{array}{c}\text { MAF } \\
\text { (ExAC) }\end{array}$ & PP2 & $\begin{array}{c}\text { SIF } \\
\text { T }\end{array}$ & MT & Disease association \\
\hline $\begin{array}{l}\text { ADAMTS1 } \\
3\end{array}$ & c. $1342 C>G$ & p.Q448E & 2301612 & 0.453 & BEN & Tol & Poly & $\begin{array}{l}\text { Congenital Thrombotic } \\
\text { Thrombocytopenic Purpura }\end{array}$ \\
\hline $\begin{array}{c}\text { ADAMTS1 } \\
3\end{array}$ & c. $3829 \mathrm{C}>\mathrm{T}$ & p.R1277W & 140450669 & 0.00005 & BEN & Tol & Poly & - \\
\hline VWF & c. $2555 \mathrm{~A}>\mathrm{G}$ & p.Q852R & 216321 & 0.1008 & BEN & Tol & Poly & $\begin{array}{c}\text { von Willebrand Disease } \\
\text { (Type 2N) }\end{array}$ \\
\hline VWF & c. $2365 \mathrm{~A}>\mathrm{G}$ & p.T789A, & 1063856 & 0.3232 & BEN & Tol & Poly & - \\
\hline VWF & c. $1451 \mathrm{~A}>\mathrm{G}$ & p.H484R, & 1800378 & 0.3788 & BEN & Tol & Poly & - \\
\hline CFB & c. $94 \mathrm{C}>\mathrm{T}$ & p.R32W & 12614 & 0.1312 & $\begin{array}{c}\text { POS } \\
\text { DAM }\end{array}$ & Del & Poly & Chronic Hepatitis B \\
\hline CFI & c. $898 \mathrm{~A}>\mathrm{G}$ & p.T300A & 11098044 & 0.0094 & BEN & Tol & Poly & - \\
\hline $\mathrm{CFH}$ & c. $1204 \mathrm{C}>\mathrm{T}$ & p.H402Y, & 1061170 & 0.3279 & BEN & Tol & Poly & $\begin{array}{c}\text { Age related macular } \\
\text { degeneration }\end{array}$ \\
\hline $\mathrm{CFH}$ & c. $2821 \mathrm{G}>\mathrm{T}$ & p.V941F, & 529492401 & 0.0004 & & & Poly & - \\
\hline
\end{tabular}

MAF- Minor allele frequency, PP2-Polyphen 2, BEN-Benign, POS DAM-Possibly Damaging, SIFT- Sorting Intolerant from Tolerant, TolTolerable, Del-Deleterious, MT-Mutation Taster, Poly-Polymorphism

ADAMTS13: a disintegrin like and metalloproteinase with thrombospondin type 1 motifs - 13; vWF: von-Willebrand factor; CFB:

Complement factor B; CFI : Complement factor I; CFH: Complement factor $\mathrm{H}$ 
Table 3 : Laboratory values of NCIPH patient with detectable R1277W variation in CUB domain of ADAMTS13 gene

\begin{tabular}{|l|l|l|l|l|}
\hline Time & Mar '14 & Jul '14 & Nov '14 & Feb '15 \\
\hline Plasma ADAMTS13-CBA (\%)* & $<10$ & 72 & 77 & 87 \\
\hline Plasma vWF-CBA (\%)* & 421 & 495 & 379 & 565 \\
\hline Platelets (X10\%/cmm) & 3.41 & 1.87 & & 1.31 \\
\hline Child's score & 8 & 6 & 6 & 7 \\
\hline Serum bilirubin (mg/dl) & 0.4 & 0.5 & 0.46 & 0.27 \\
\hline Serum albumin (mg/dl) & 1.5 & 2.8 & 3.1 & 2.2 \\
\hline MELD score & 13 & 13 & & 15 \\
\hline *Normal ranges - ADAMTS13-CBA: 50\%-150\%; vWF-CBA: 50\%-160\% & & & \\
\hline
\end{tabular}

ADAMTS13: a disintegrin like and metalloproteinase with thrombospondin type 1 motifs - 13; vWF: von-Willebrand factor; CBA: Collagen binding activity assay; MELD : Model for end-stage liver disease 

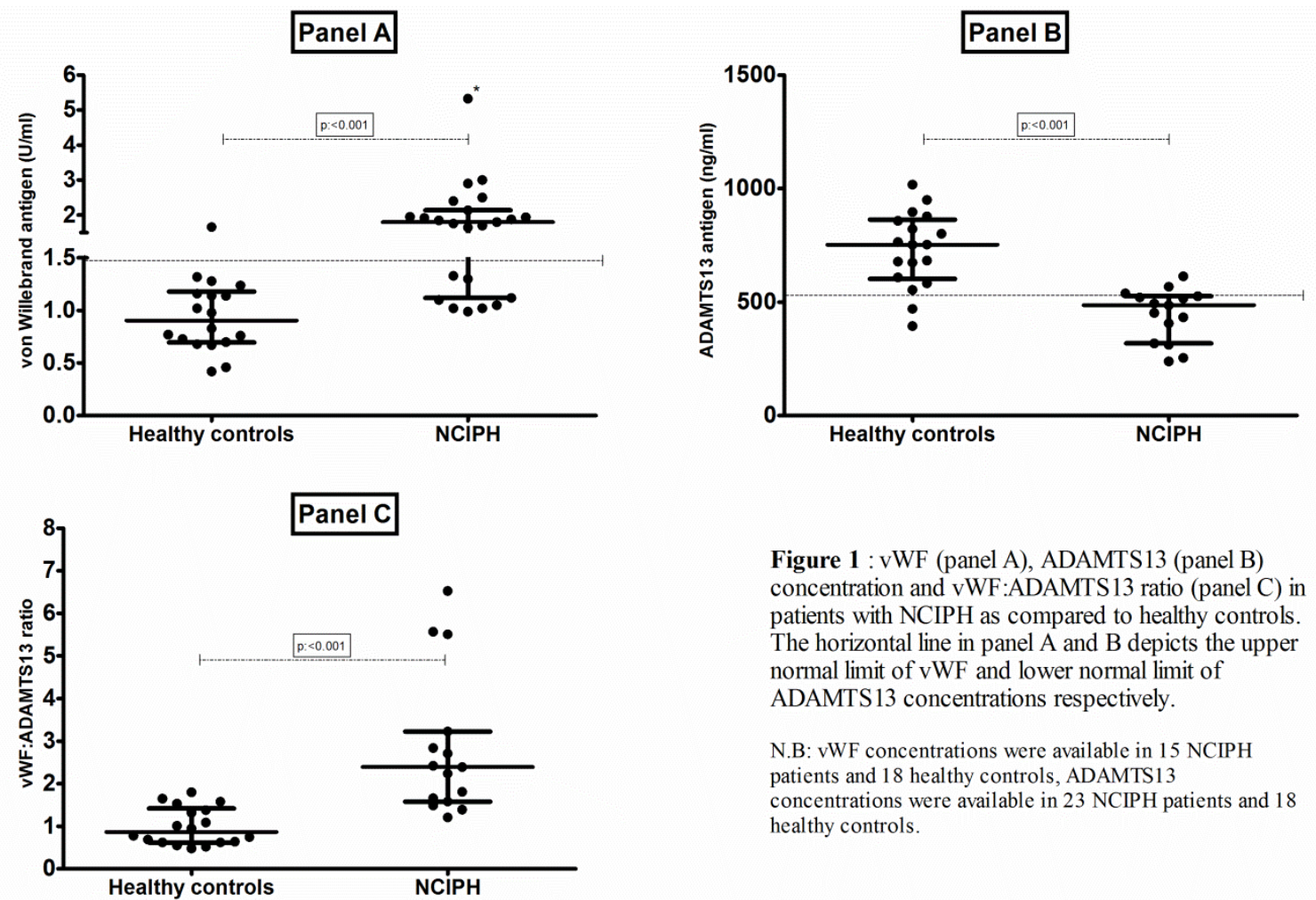

Figure 1 : vWF (panel A), ADAMTS13 (panel B) concentration and vWF:ADAMTS13 ratio (panel C) in patients with NCIPH as compared to healthy controls. The horizontal line in panel A and $\mathrm{B}$ depicts the upper normal limit of vWF and lower normal limit of ADAMTS 13 concentrations respectively.

N.B: vWF concentrations were available in $15 \mathrm{NCIPH}$ patients and 18 healthy controls, ADAMTS13

concentrations were available in $23 \mathrm{NCIPH}$ patients and 18 healthy controls. 


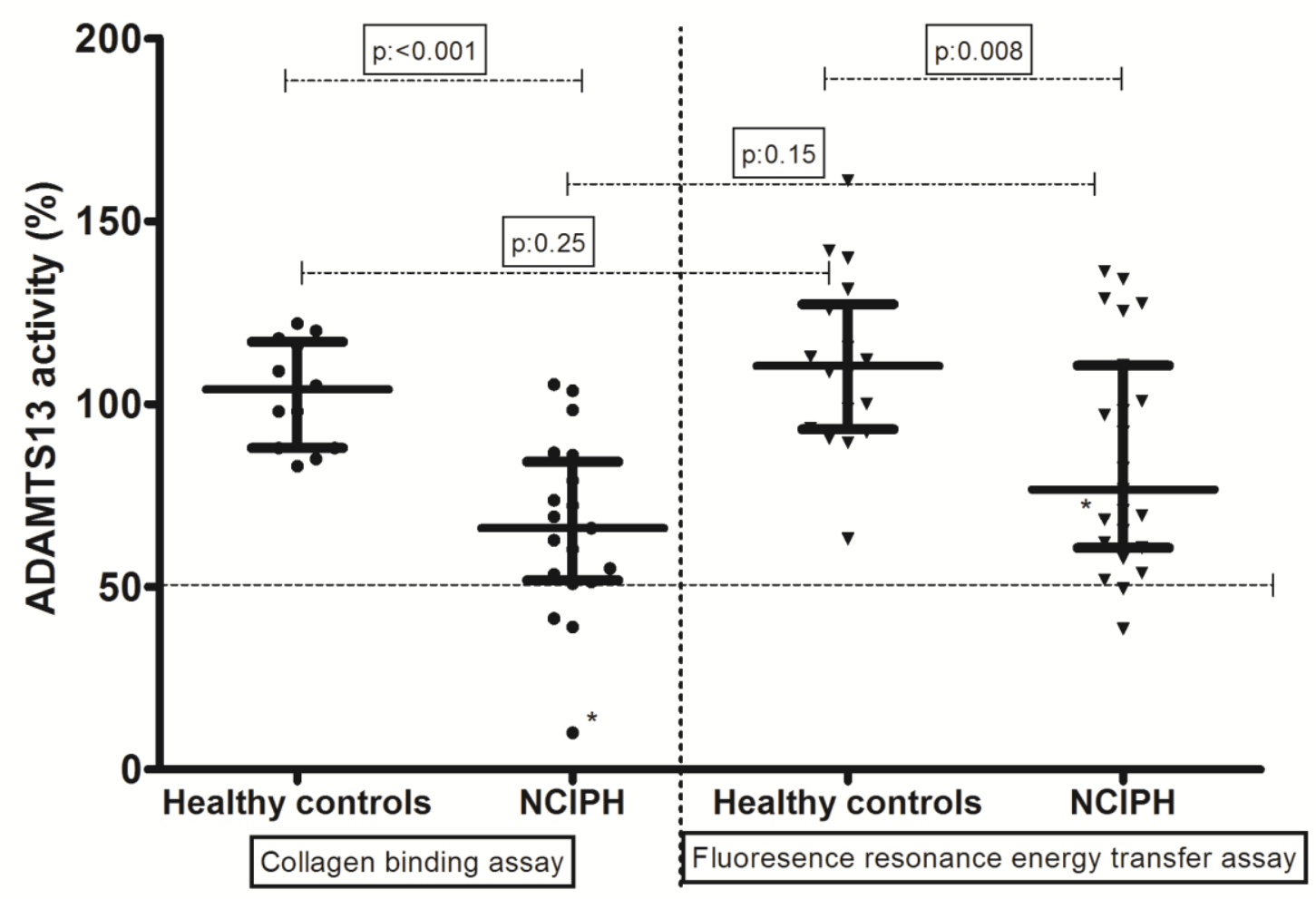

Figure 2 : Plasma ADAMTS13 activity assayed by FRET (fluorescence resonance energy transfer) assay and collagen binding assay (CBA) in NCIPH patients as compared to healthy controls. The ADAMTS13 values for the patient with R1227W mutation in ADAMTS13 CUB domain are marked with asterisk $(*)$.

N.B: FRET available in 23 NCIPH patients and 18 healthy controls, CBA available in 20 NCIPH patients and 13 healthy controls. 

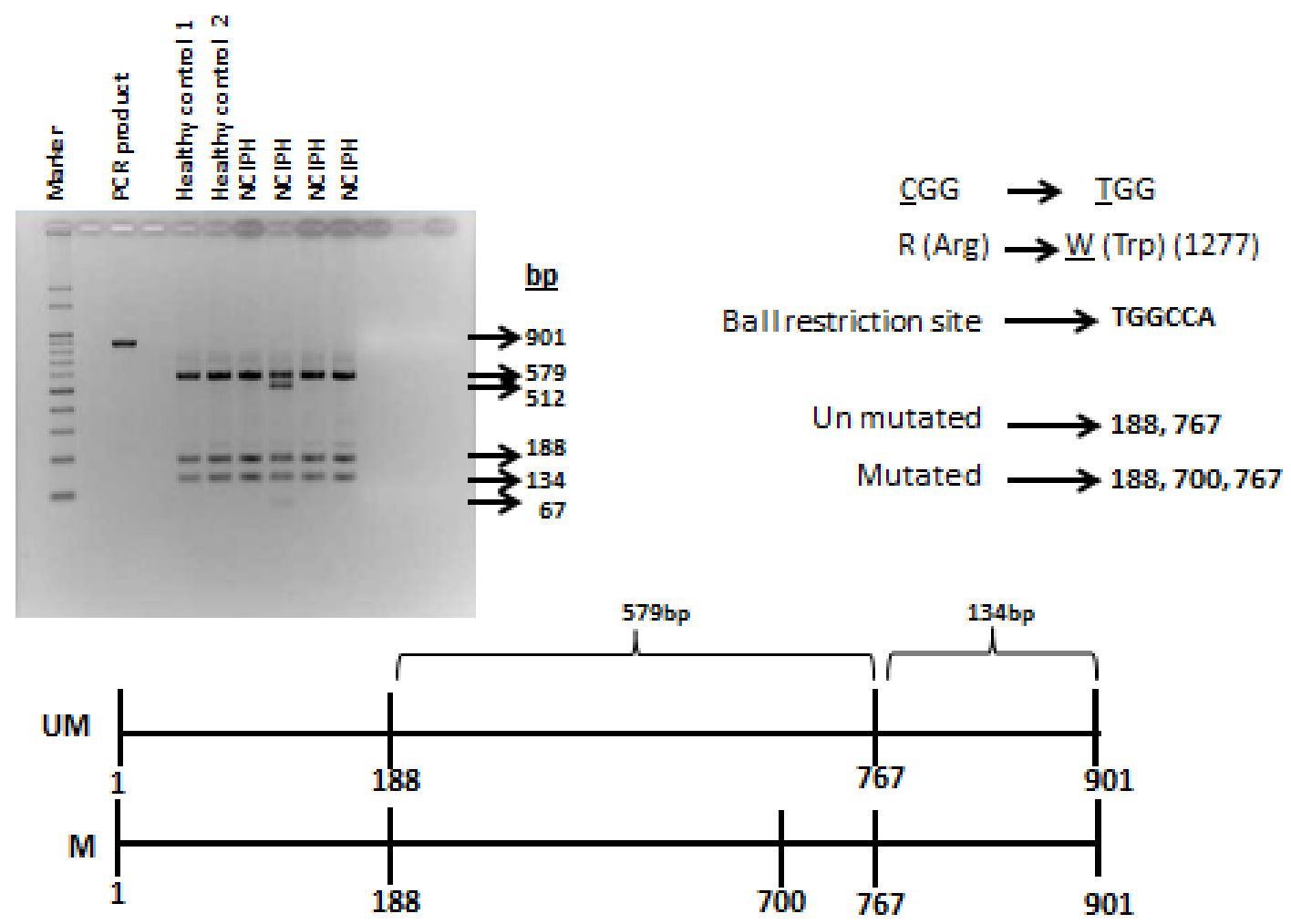

Figure 3: Restriction fragment length polymorphism on the ADAMTS13 CUB domain PCR product (901 bp). The mutation R1227W created a new restriction site for DNA restriction enzyme BalI, resulting in extra bands in one NCIPH patient 

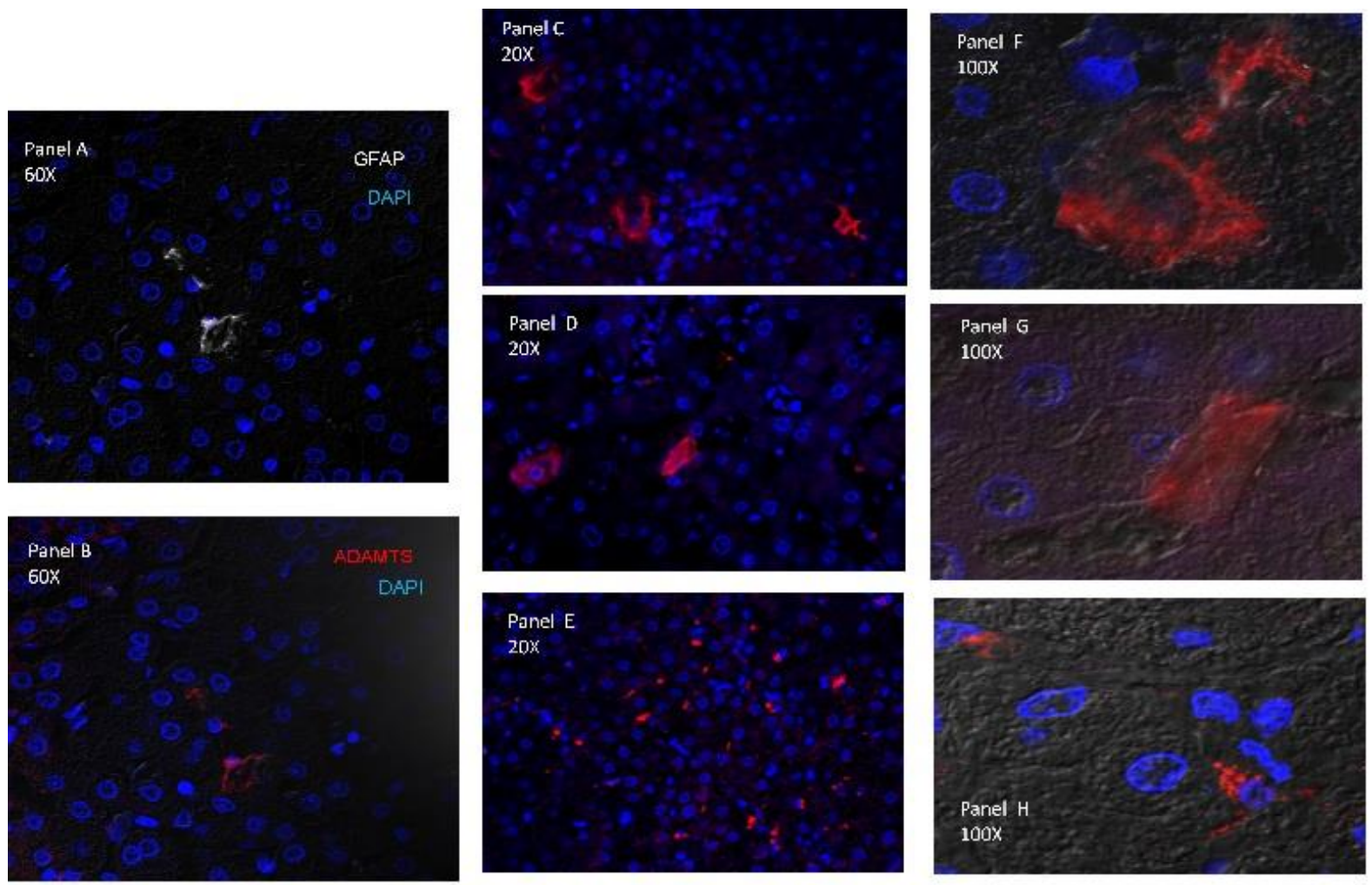

Figure 4 : Indirect immunofluorescence of liver biopsy. Panel A and B shown co-localisation of stellate cell marker GFAP (white) and ADAMTS13 (red) in normal liver. Panels C and F (normal), panels D and G (cirrhotic control) and panels E and H (NCIPH patient with R1277W mutation) show more diffuse and punctate staining as well as ADAMTS13 globules in the higher magnification in NCIPH patient. Similar globules were not seen in normal/ cirrhotic liver biopsy. 


\section{References}

1. Goel A, Ramakrishna B, Madhu K, Zachariah U, Ramachandran J, Keshava SN, Elias E, Eapen CE. Idiopathic noncirrhotic intrahepatic portal hypertension is an ongoing problem in India. Hepatology 2011;54:2274; author reply 2274-5.

2. Goel A, Madhu K, Zachariah U, Sajith KG, Ramachandran J, Ramakrishna B, Gibikote S, Jude J, Chandy GM, Elias E, Eapen CE. A study of aetiology of portal hypertension in adults (including the elderly) at a tertiary centre in southern India. Indian J Med Res 2013;137:922-7.

3. Goel A, Alagammai PL, Nair SC, Mackie I, Ramakrishna B, Muliyil J, Keshava SN, Eapen CE, Elias E. ADAMTS13 deficiency, despite well-compensated liver functions in patients with noncirrhotic portal hypertension. Indian J Gastroenterol 2014;33:35563.

4. Mackie I, Eapen CE, Neil D, Lawrie AS, Chitolie A, Shaw JC, Elias E. Idiopathic noncirrhotic intrahepatic portal hypertension is associated with sustained ADAMTS13 Deficiency. Dig Dis Sci 2011;56:2456-65.

5. Uemura M, Tatsumi K, Matsumoto M, Fujimoto M, Matsuyama T, Ishikawa M, Iwamoto TA, Mori T, Wanaka A, Fukui H, Fujimura Y. Localization of ADAMTS13 to the stellate cells of human liver. Blood 2005;106:922-4.

6. Lancellotti S, De Cristofaro R. Structure and proteolytic properties of ADAMTS13, a metalloprotease involved in the pathogenesis of thrombotic microangiopathies. Prog Mol Biol Transl Sci 2011;99:105-44.

7. Zhou Z, Yeh HC, Jing H, Wang C, Tao Z, Choi H, Aboulfatova K, Li R, Dong JF. Cysteine residues in CUB-1 domain are critical for ADAMTS13 secretion and stability. Thromb Haemost 2011;105:21-30. 
8. Pimanda JE, Maekawa A, Wind T, Paxton J, Chesterman CN, Hogg PJ. Congenital thrombotic thrombocytopenic purpura in association with a mutation in the second CUB domain of ADAMTS13. Blood 2004;103:627-9.

9. Turner NA, Moake J. Assembly and activation of alternative complement components on endothelial cell-anchored ultra-large von Willebrand factor links complement and hemostasis-thrombosis. PLoS One 2013;8:e59372.

10. Tati R, Kristoffersson AC, Stahl AL, Rebetz J, Wang L, Licht C, Motto D, Karpman D. Complement activation associated with ADAMTS13 deficiency in human and murine thrombotic microangiopathy. J Immunol 2013;191:2184-93.

11. Koot BG, Alders M, Verheij J, Beuers U, Cobben JM. A de novo mutation in KCNN3 associated with autosomal dominant idiopathic non-cirrhotic portal hypertension. J Hepatol 2016;64:974-7.

12. Vilarinho S, Sari S, Yilmaz G, Stiegler AL, Boggon TJ, Jain D, Akyol G, Dalgic B, Gunel M, Lifton RP. Recurrent recessive mutation in deoxyguanosine kinase causes idiopathic noncirrhotic portal hypertension. Hepatology 2016;63:1977-86.

13. Gerritsen HE, Turecek PL, Schwarz HP, Lammle B, Furlan M. Assay of von Willebrand factor (vWF)-cleaving protease based on decreased collagen binding affinity of degraded vWF: a tool for the diagnosis of thrombotic thrombocytopenic purpura (TTP). Thromb Haemost 1999;82:1386-9.

14. Eura Y, Kokame K, Takafuta T, Tanaka R, Kobayashi H, Ishida F, Hisanaga S, Matsumoto M, Fujimura Y, Miyata T. Candidate gene analysis using genomic quantitative PCR: identification of ADAMTS13 large deletions in two patients with Upshaw-Schulman syndrome. Mol Genet Genomic Med 2014;2:240-4.

15. Kokame K, Miyata T. Genetic defects leading to hereditary thrombotic thrombocytopenic purpura. Semin Hematol 2004;41:34-40. 
16. Kokame K, Kokubo Y, Miyata T. Polymorphisms and mutations of ADAMTS13 in the Japanese population and estimation of the number of patients with UpshawSchulman syndrome. J Thromb Haemost 2011;9:1654-6.

17. Arning A, Jeibmann A, Kohnemann S, Brokinkel B, Ewelt C, Berger K, Wellmann J, Nowak-Gottl U, Stummer W, Stoll M, Holling M. ADAMTS genes and the risk of cerebral aneurysm. J Neurosurg 2016;125:269-74.

18. Reuken PA, Kussmann A, Kiehntopf M, Budde U, Stallmach A, Claus RA, Bruns T. Imbalance of von Willebrand factor and its cleaving protease ADAMTS13 during systemic inflammation superimposed on advanced cirrhosis. Liver Int 2015;35:37-45.

19. Takaya H, Uemura M, Fujimura Y, Matsumoto M, Matsuyama T, Kato S, Morioka C, Ishizashi H, Hori $\mathrm{Y}$, Fujimoto $\mathrm{M}$, Tsujimoto $\mathrm{T}$, Kawaratani H, Toyohara M, Kurumatani N, Fukui H. ADAMTS13 activity may predict the cumulative survival of patients with liver cirrhosis in comparison with the Child-Turcotte-Pugh score and the Model for End-Stage Liver Disease score. Hepatol Res 2012;42:459-72.

20. Uemura M, Fujimura Y, Matsumoto M, Ishizashi H, Kato S, Matsuyama T, Isonishi A, Ishikawa M, Yagita M, Morioka C, Yoshiji H, Tsujimoto T, Kurumatani N, Fukui H. Comprehensive analysis of ADAMTS13 in patients with liver cirrhosis. Thromb Haemost 2008;99:1019-29.

21. Sidore C, Busonero F, Maschio A, Porcu E, Naitza S, Zoledziewska M et al. Genome sequencing elucidates Sardinian genetic architecture and augments association analyses for lipid and blood inflammatory markers. Nat Genet 2015;47:1272-81.

22. Park JH, Gail MH, Weinberg CR, Carroll RJ, Chung CC, Wang Z, Chanock SJ, Fraumeni JF, Jr., Chatterjee N. Distribution of allele frequencies and effect sizes and their interrelationships for common genetic susceptibility variants. Proc Natl Acad Sci U S A 2011;108:18026-31. 
23. Fan X, Kremer Hovinga JA, Shirotani-Ikejima H, Eura Y, Hirai H, Honda S, Kokame K, Taleghani MM, von Krogh AS, Yoshida Y, Fujimura Y, Lammle B, Miyata T. Genetic variations in complement factors in patients with congenital thrombotic thrombocytopenic purpura with renal insufficiency. Int J Hematol 2016;103:283-91.

24. De Cock E, Hermans C, De Raeymaecker J, De Ceunynck K, De Maeyer B, Vandeputte N, Vandenbulcke A, Deckmyn H, Rottensteiner H, De Maeyer M, De Meyer SF, Vanhoorelbeke K. The novel ADAMTS13-p.D187H mutation impairs ADAMTS13 activity and secretion and contributes to thrombotic thrombocytopenic purpura in mice. J Thromb Haemost 2015;13:283-92.

25. Perez-Rodriguez A, Loures E, Rodriguez-Trillo A, Costa-Pinto J, Garcia-Rivero A, Batlle-Lopez A, Batlle J, Lopez-Fernandez MF. Inherited ADAMTS13 deficiency (Upshaw-Schulman syndrome): a short review. Thromb Res 2014;134:1171-5.

26. Camilleri RS, Scully M, Thomas M, Mackie IJ, Liesner R, Chen WJ, Manns K, Machin SJ. A phenotype-genotype correlation of ADAMTS13 mutations in congenital thrombotic thrombocytopenic purpura patients treated in the United Kingdom. J Thromb Haemost 2012;10:1792-801

27. Schwameis M, Schorgenhofer C, Assinger A, Steiner MM, Jilma B. VWF excess and ADAMTS13 deficiency: a unifying pathomechanism linking inflammation to thrombosis in DIC, malaria, and TTP. Thromb Haemost 2015;113:708-18.

28. Sonneveld MA, de Maat MP, Leebeek FW. Von Willebrand factor and ADAMTS13 in arterial thrombosis: a systematic review and meta-analysis. Blood Rev 2014;28:167-78.

29. Kokame K. [Genetic polymorphism of von Willebrand factor (VWF)-cleaving protease, ADAMTS13]. Brain Nerve 2008;60:1325-32. 
30. Feng S, Eyler SJ, Zhang Y, Maga T, Nester CM, Kroll MH, Smith RJ, AfsharKharghan V. Partial ADAMTS13 deficiency in atypical hemolytic uremic syndrome. Blood 2013;122:1487-93.

31. Edwards NC, Hing ZA, Perry A, Blaisdell A, Kopelman DB, Fathke R et al. Characterization of coding synonymous and non-synonymous variants in ADAMTS13 using ex vivo and in silico approaches. PLoS One 2012;7:e38864.

32. Lotta LA, Tuana G, Yu J, Martinelli I, Wang M, Yu F, Passamonti SM, Pappalardo E, Valsecchi C, Scherer SE, Hale Wt, Muzny DM, Randi G, Rosendaal FR, Gibbs RA, Peyvandi F. Next-generation sequencing study finds an excess of rare, coding singlenucleotide variants of ADAMTS13 in patients with deep vein thrombosis. J Thromb Haemost 2013;11:1228-39.

33. Goel A, Elias JE, Eapen CE, Ramakrishna B, Elias E. Idiopathic Non-Cirrhotic Intrahepatic Portal Hypertension (NCIPH)-Newer Insights into Pathogenesis and Emerging Newer Treatment Options. J Clin Exp Hepatol 2014;4:247-56.

34. Maiwall R, Goel A, Pulimood AB, Babji S, Sophia J, Prasad C et al. Investigation into celiac disease in Indian patients with portal hypertension. Indian J Gastroenterol 2014;33:517-23.

35. Goel A, Christudoss P, George R, Ramakrishna B, Amirtharaj GJ, Keshava SN et al. Arsenicosis, possibly from contaminated groundwater, associated with noncirrhotic intrahepatic portal hypertension. Indian J Gastroenterol 2016;35:207-15.

36. Goel A, Ramakrishna B, Muliyil J, Madhu K, Sajith KG, Zachariah U et al. Use of serum vitamin B12 level as a marker to differentiate idiopathic noncirrhotic intrahepatic portal hypertension from cryptogenic cirrhosis. Dig Dis Sci 2013;58:17987. 
37. Goel A, Ramakrishna B, Job V, Zachariah U, Eapen CE. Vitamin B12 Deficiency in Patients With Unexplained Portal Hypertension Indicates Absence of Severe Liver Fibrosis. J Clin Gastroenterol. Publish Ahead of Print.

38. Wanless IR, Wong F, Blendis LM, Greig P, Heathcote EJ, Levy G. Hepatic and portal vein thrombosis in cirrhosis: possible role in development of parenchymal extinction and portal hypertension. Hepatology 1995;21:1238-47.

39. Goel A, Amirtharaj GJ, Sarvanan S, Ramakrishna B, Ramachandran A, Balasubramanian KA, Zachariah U, Sajith KG, Mackie I, Elias E, Eapen CE. ADAMTS13 Deficiency Predisposes to Systemic Macro and Microvascular Complications in NCIPH Patients. J Clin Exp Hepatol 2016;6:S100.

40. Eerenberg ES, Levi M. The potential therapeutic benefit of targeting ADAMTS13 activity. Semin Thromb Hemost 2014;40:28-33. 